\title{
L'ecografia nella diagnostica delle patologie genito-urinarie
}

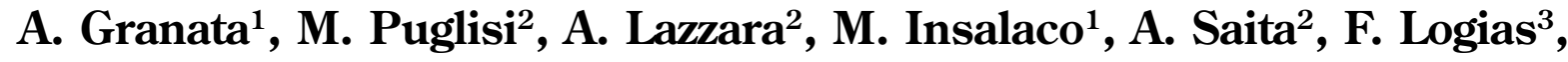

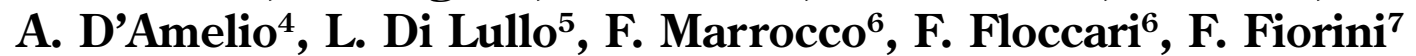 \\ ${ }^{1}$ U.O.C. Nefrologia e Dialisi, Ospedale S. Giovanni di Dio, Agrigento \\ ${ }^{2}$ Dip. di Nefrologia-Dialisi ed Urologia, AOU "Policlinico Vittorio Emanuele”, Catania \\ ${ }^{3}$ U.O.C. Nefrologia e Dialisi, Ospedale "S. Camillo", Sorgono \\ ${ }^{4}$ U.O.C. Nefrologia, Dialisi e Trapianto, Ospedale "V. Fazzi”, Lecce \\ ${ }^{5}$ U.O.C. Nefrologia e Dialisi, Ospedale "L. Parodi Delfino", Colleferro \\ ${ }^{6}$ U.O.C. Nefrologia e Dialisi, Ospedale "S. Paolo", Civitavecchia \\ ${ }^{7}$ U.O.C. Nefrologia e Dialisi, Ospedale S. Maria della Misericordia, Rovigo
}

ULTRASOUND IN THE GENITO-URINARY PATHOLOGIES

ABstract. Urinary tract infections are a frequent clinical problem in all ages. Ultrasound is a valuable tool in the diagnosis and management of infections, allowing you to quickly diagnose complications and predisposing factors.

This review provides an overview of the principal sonographic pictures during infections of bladder, prostate, seminal vesicles, testicles, epididymis and scrotum, providing a handy quick reference tool to the nephrologist interested in ultrasonography.

KeY WoRDs. Ultrasonography, Urinary tract infections, Cystitis, Prostatitis, Orchytis

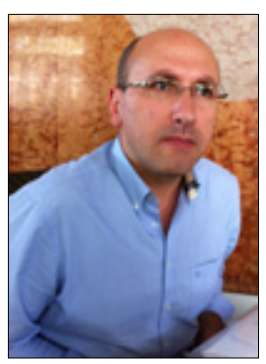

Antonio Granata

\section{Introduzione}

Le infezioni delle vie urinarie (IVU) costituiscono un problema clinico frequente in tutte le età. Si stima che negli USA da 5 a 6 milioni di visite mediche ogni anno siano dovute a infezioni delle vie urinarie (IVU) (1).

L'IVU rappresenta una delle infezioni più comuni, con una incidenza nettamente più elevata nella popolazione femminile con almeno un episodio sintomatico l'anno nel 25-35\% delle donne di età compresa tra i 20 e i 40 anni (1). Con il progredire dell'età anagrafica, la differenza nell'incidenza tra i due sessi va assottigliandosi, pur rimanendo più elevata nel sesso femminile anche nella popolazione dei grandi anziani (1).

Dal punto di vista epidemiologico, le IVU più comuni sono rappresentate dalle cistiti, mentre viene riportata un'incidenza minore di uretriti, cistopieliti e pielonefriti. Tutte queste patologie sono prevalentemente legate ad infezioni batteriche da gram negativi, di provenienza intestinale (Escherichia Coli, Proteus Spp, Enterococcus $S p p$ come agenti più frequentemente isolati), seppure siano frequenti anche le infezioni da Chlamydiae e non del tutto eccezionali le infezioni da Gram+ e miceti (1). Le IVU possono essere complicate dalla presenza di ostruzione delle vie urinarie, nefrolitiasi, reflusso vescico-ureterale, setticemia, diabete mellito, malattie sistemiche connesse con condizioni di immunodeficienza (insufficienza renale cronica, AIDS, linfomi, leucemie ecc...).

Esente dall'utilizzo di radiazioni ionizzanti, dalla potenziale nefrotossicità dei mezzi di contrasto iodati e dalle problematiche legate al gadolinio, l'esame ultrasonografico risulta di grande utilità: 
- nell'escludere che l'IVU sia accompagnata da patologie urinarie preesistenti o sopraggiunte (idronefrosi, cisti infette, rene policistico dell'adulto, neoplasie ecc...);

- nellidentificare il grado di eventuale interessamento del parenchima renale (pielonefrite diffusa, focale) ovvero la presenza di essudato purulento (ascesso renale, para-renale, pielonefrite enfisematosa e xanto-granulomatosa);

- nellindividuare patterns ecografici compatibili con la presenza di infezioni croniche del parenchima renale (scarring, alterazioni dell'ecogenicità del profilo renale).

Lo studio ecografico delle basse vie urinarie, non sempre "nelle corde" del nefrologo ecografista, può offrire valide informazioni cliniche in corso di IVU. Scopo della presente trattazione sarà quindi offrire una rapida disamina delle possibili applicazioni dell'ecografia nella diagnostica delle infezioni delle basse vie urinarie.

\section{L'ecografia nella valutazione delle infezioni della vescica}

Lo studio ecografico della vescica può essere condotto per via sovra-pubica ovvero, tramite sonde endo-cavitarie, per via trans-rettale, trans-vaginale e trans-uretrale. Lo studio sovra-pubico rappresenta la metodica di prima istanza e deve sempre precedere gli eventuali approcci di secondo livello ai quali si dovrà ricorrere solo nel caso in cui si sospetti la presenza di patologie altrimenti non diagnosticabili (ad esempio, patologie del collo della vescica).

Per lo studio sovra-pubico si impiegano sonde convex da 3.5-5.0 MHz, le quali consentono la visualizzazione completa della vescica e degli organi adiacenti. Trasduttori ad elevata frequenza $5.0-7.5 \mathrm{MHz}$ vengono impiegati per lo studio della cupola e della parete anteriore della vescica.

L'esame va condotto a vescica piena e concluso con scansioni in fase post-minzionale, al fine di poter escludere o quantificare l'eventuale residuo post-minzionale. Il grado di riempimento vescicale deve essere adeguato ma non eccessivo. Se, infatti, la scarsa distensione riduce la possibilità di discriminare eventuali anomalie di parete, anche l'eccessivo riempimento può dar luogo ad artefatti (vedi falsi aspetti di ectasia delle vie urinarie), ovvero attenuare eventuali irregolarità delle pareti (2). Un buon metro di misura del grado di riempimento della vescica è la capacità del paziente di percepire lo stimolo ad urinare nitidamente, senza che questo divenga imperioso ed intollerabile. Nel paziente cateterizzato è bene ricordarsi di bloccare il deflusso delle urine nei 30-40' precedenti l'esame, evitando per quanto possibile di riempire in maniera retrograda la vescica.

La vescica viene studiata con scansioni eseguite lungo un piano longitudinale (che permette di esaminare la parete posteriore, il trigono e la regione retro trigonale), trasversale (ideale per esaminare le pareti laterali e definirne i rapporti con gli organi circostanti) e obliquo (utile a ottimizzare l'immagine degli orifizi ureterali e del tratto distale degli ureteri).

Di particolare importanza è il saper distinguere eventuali artefatti, strettamente correlati alla tecnica ecografica: dalle ombre acustiche laterali, all'effetto pioggia, all'artefatto dei lobi laterali. Questi artefatti possono essere ridotti o addirittura eliminati mutando la posizione della sonda, insonando l'organo perpendicolarmente, ovvero riducendo il guadagno e utilizzando l'armonica tissutale (o seconda armonica) (3).

\section{La cistite acuta}

I segni ecografici più salienti sono rappresentati dall'ispessimento diffuso o focale (collo e trigono) della parete, espressione di edema, da echi grossolani in sospensione nel contenuto vescicale o da immagini di sedimento in relazione a detriti flogistici, pus e sangue $(4,5)$.

La vescica può apparire armonicamente piccola a causa dell'ipertonia vescicale riflessa che determina una ridotta distensione della stessa.

La cistite enfisematosa (6) è una rara entità clinica, caratterizzata dalla formazione di piccole vescicole piene di gas nella mucosa e nella sottomucosa che all'esame ultrasonografico si presentano come aree iperecogene intrappolate che ostacolano la trasmissione del fascio ultrasonoro. La presenza di pneumaturia deve far sospettare la presenza di fistole entero-vescicali, che vanno correttamente indagate con ulteriori indagini strumentali, radiologiche ed endoscopiche.

In caso di cistiti acute ricorrenti (7), oltre ai comuni fattori di rischio (stipsi cronica, diabete mellito, scarsa igiene, presenza di cateteri vescicali ovvero altri presidi di continenza, incontinenza urinaria ed intensa attività sessuale), occorre ricercare sistematicamente la presenza di condizioni predisponenti apprezzabili con la metodica, quali diverticoli vescicali, residuo post-minzionale, litiasi, trabecolatura da sforzo.

\section{Le cistiti croniche}

I processi infiammatori cronici focali della vescica si ma- 


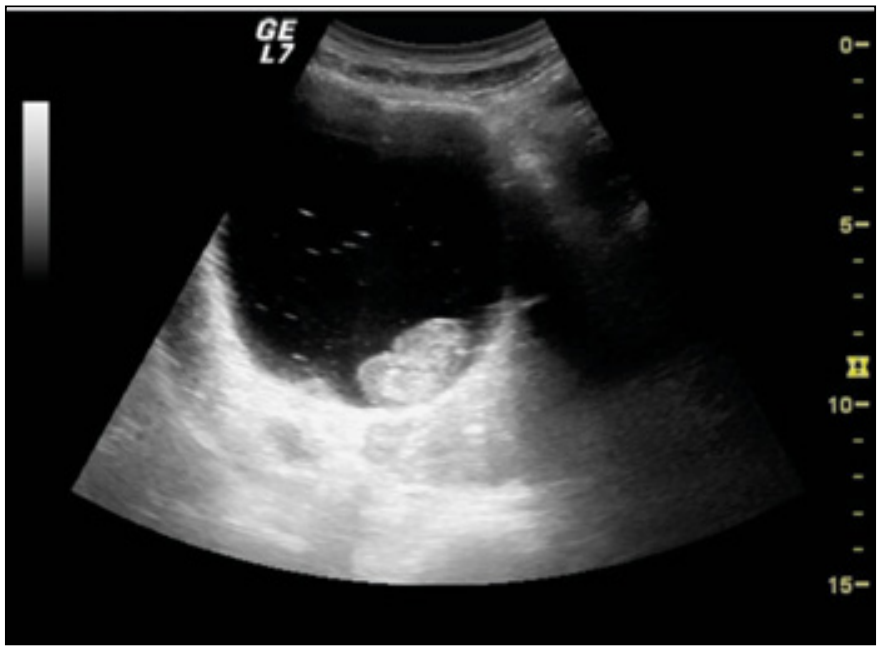

Fig. 1 - Voluminosa formazione iperecogena apparentemente adesa al pavimento vescicale, in paziente macroematurico. La massa varia posizione al variare del decubito e si lascia aspirare dopo cateterismo, risultando essere un voluminoso coagulo.

nifestano sotto forma di ispessimento circoscritto della parete con aspetto pseudo tumorale. In questo contesto rientra la cistite bollosa, il cui quadro ecografico è caratterizzato da un ispessimento ipoecogeno della mucosa, apparentemente privo di asperità nelle fasi iniziali ma con pattern polipoide nelle fasi avanzate; tale ispessimento si presenta, generalmente, localizzato a livello della parete posteriore ovvero diffuso a tutta la parete vescicale nelle situazioni più inveterate (8).

Il prolungato stazionamento dei cateteri vescicali rappresenta la causa più frequente delle forme di cistite appena descritte (9). La cistite attinica, associata alla radioterapia, comporta invece assottigliamento o scomparsa della tunica muscolare con inspessimento della mucosasottomucosa; in questi casi le pareti della vescica appaiono estremamente esili. In corso di schistosomiasi sarà possibile invece rilevare la presenza di calcificazioni parietali, localizzate a livello della tunica muscolare e della sottomucosa (10).

\section{Prostata e vescicole seminali}

L'approccio addominale alla prostata e alle vescicole seminali avviene utilizzando sonde convesse da 3.5-5.0 $\mathrm{Mhz}$, con scansioni portate sulla parete addominale, immediatamente al di sopra della sinfisi pubica. La sonda viene angolata verso il basso, eseguendo scansioni longitudinali, trasversali e oblique. E possibile in tal modo determinare il volume della ghiandola, la cui misura

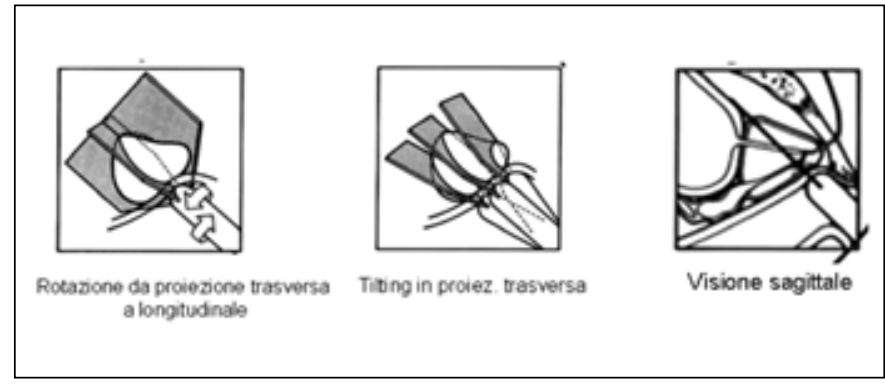

Fig. 2 - Scansioni utili allo studio della prostata (modificato da Torp-Pedersen ST, Lee F. Transrectal biopsy of the prostate guided by transrectal ultrasound. Urol Clin N Am 1989;16(4): 703-12).

seriata nel tempo può essere estremamente utile nella gestione della terapia delle prostatiti e delle neoplasie prostatiche, così come nel fornire indicazioni all'approccio chirurgico.

Il volume prostatico viene calcolato secondo la formula dell'ellissoide, moltiplicando i tre diametri principali per una costante ( $\mathrm{L} \times \mathrm{T} \times \mathrm{V} \times \mathrm{0.523}$ ). Il volume così ottenuto presenta una buona accuratezza, con errore del $10 \%$ circa, del tutto accettabile nella pratica clinica. La volumetria prostatica normale in un uomo di 20-30 anni è 20 +/- $6 \mathrm{cc}$, ma è destinata ad aumentare con l'ipertrofia prostatica benigna (IPB).

Il peso prostatico può essere invece calcolato moltiplicando il volume calcolato per una costante pari a 1.06 (peso specifico del tessuto ghiandolare).

La valutazione del volume prostatico consente la determinazione del PSA density, ossia il rapporto tra PSA sierico e volume prostatico. Un valore di PSA density $>=0.15$ viene

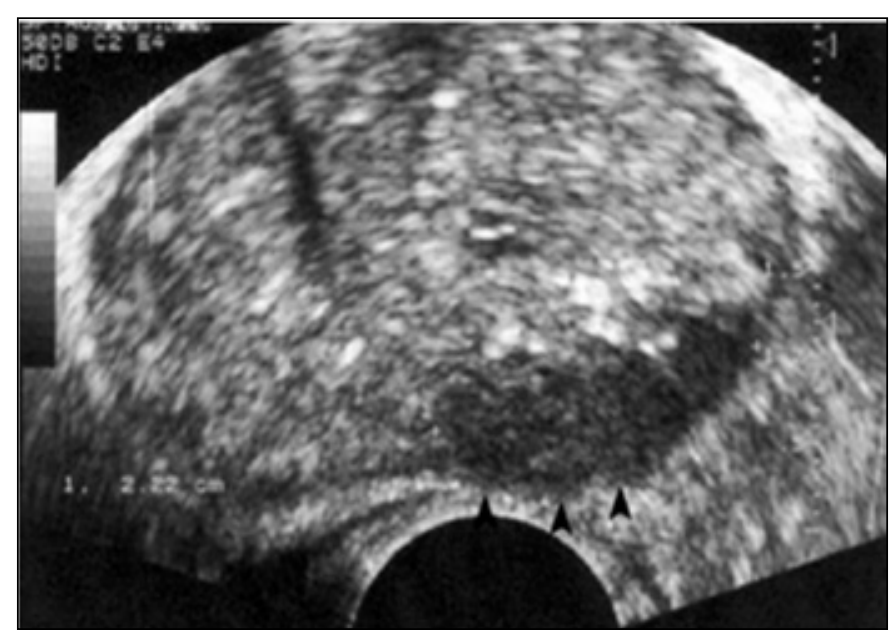

Fig. 3 - Scansione trans-rettale. Lesione prostatica ipoecogena periferica da carcinoma prostatico. 


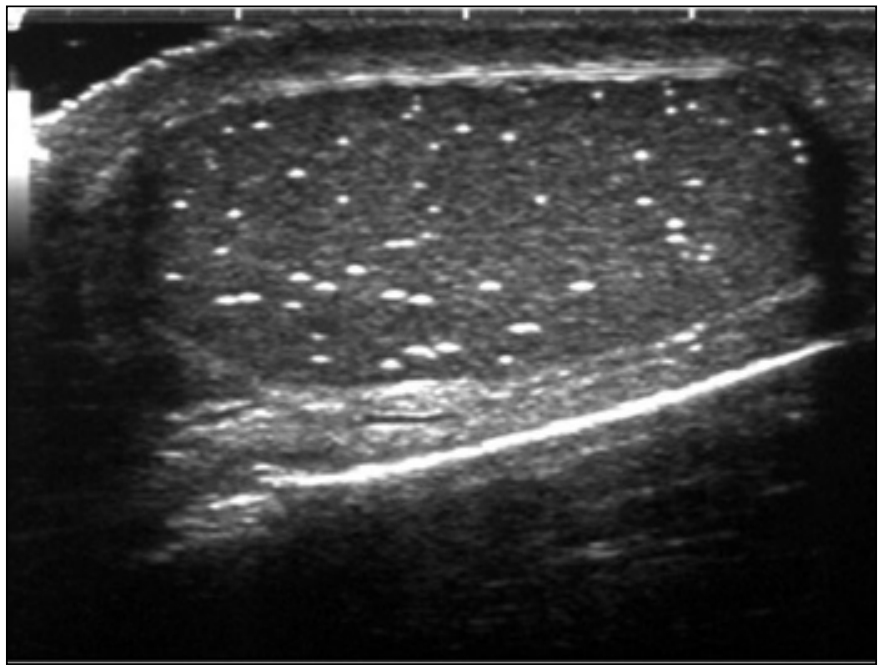

Fig. 4 - Scansione sagittale del didimo. Multipli spots iperecogeni disseminati lungo tutto il testicolo, da microlitiasi testicolare, scansione longitudinale.

considerato sospetto per neoplasia prostatica (11).

Nelle flogosi prostatiche, sia acute che croniche, l'ecografia sovra-pubica può evidenziare un incremento del volume della ghiandola con una concomitante riduzione dell'ecogenicità.

Uno studio dettagliato della ghiandola prostatica deve avvalersi di proiezioni dedicate, quali l'approccio perineale, trans-uretrale e/o trans-rettale. Quest'ultimo comporta minor rischio di complicanze rispetto all'approccio trans-uretrale ed è più accurato ai fini diagnostici; può essere eseguito orientando la sonda ecografica endo-cavitaria, con frequenza compresa tra 6 e $8 \mathrm{Mhz}$, in senso longitudinale o trasversale. I margini laterali e le asimmetrie della ghiandola sono più evidenti in scansione trasversale, mentre, per lo studio della base e dell'apice, è preferibile la scansione longitudinale.

L'approccio trans-rettale è classicamente utilizzato per l'esecuzione di biopsie della prostata. La biopsia prostatica a sestanti, descritta da Hodge e colleghi nel 1989, viene considerata come il precursore della tecnica attualmente praticata (12). In generale, la tecnica transrettale, rispetto alla trans-perineale, offre il vantaggio di richiedere un percorso più breve per l'ago bioptico ed espone il paziente a un rischio infettivo minore.

La biopsia prostatica è più facilmente eseguibile con l'orientamento longitudinale della sonda, anche se l'orientamento trasversale è anch'esso impiegato con successo. Una volta introdotta la sonda ecografica nel retto, l'apice prostatico rappresenta il primo punto di repere ecografico.

La prostata normale mostra echi di livello basso-mode-

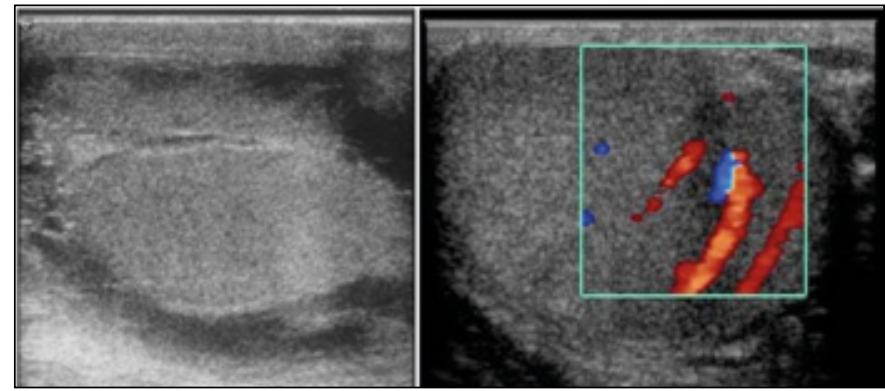

Fig. 5 - Voluminosa area ipoecogena intratesticolare da seminoma, scansione longitudinale.

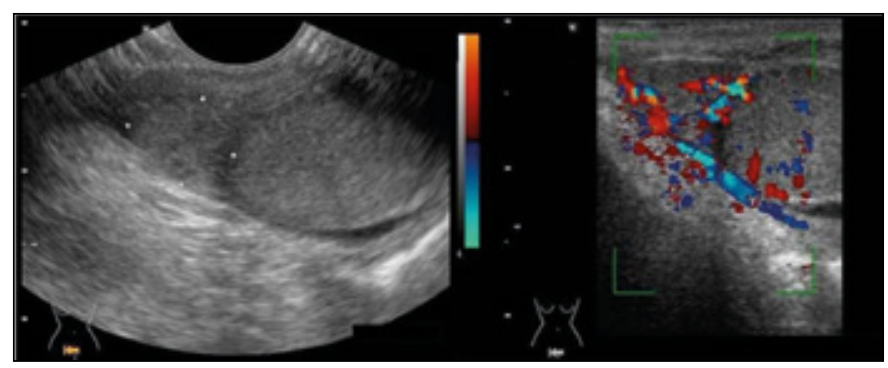

Fig. 6 - Ecografia scrotale, con sonda convex, su piano trasverso. Assenza di flusso al testicolo destro da torsione del funicolo (MAJ Robert A. Jesinger, 2009).

rato, mentre capsula prostatica e grasso peri-capsulare adiacente si presentano fortemente iper-ecogeni. La porzione peri-uretrale si presenta, solitamente, ipoecogena, mentre, in scansione longitudinale è possibile apprezzare il profilo uretrale, iper-ecogeno rispetto al parenchima prostatico. I dotti eiaculatori, si presentano sotto forma di una stria doppia e sottile, ipo-ecogena, diretta obliquamente verso le vescicole seminali. Tra prostata e parete rettale è visibile una porzione di spessore minimo, iper-ecogena, denominata fascia di Denonvilliers, mentre sono molto frequenti aree caratterizzate da ecogenicità non uniforme. Le neoformazioni benigne si presentano di solito ben definite, iper-ecogene (similmente all'aspetto della capsula prostatica), ipo-ecogene ovvero ad ecogenicità mista; i margini nodulari sono più facilmente approcciabili in scansione radiale. La presenza di ombra acustica, più facilmente evidenziabile con le scansioni lineari rispetto a quelle radiali, fornisce, solitamente, indizi di lesione benigna (13).

Indovate nel tessuto prostatico, si possono talora identificare anche cisti anecogene ripiene di liquido, che presentano un aspetto ecografico di rinforzo acustico allinterno della ghiandola (soprattutto in scansione longitudinale) orientato verso la sinfisi pubica $(12,13)$.

L'ascesso prostatico può presentarsi invece con aspetto 


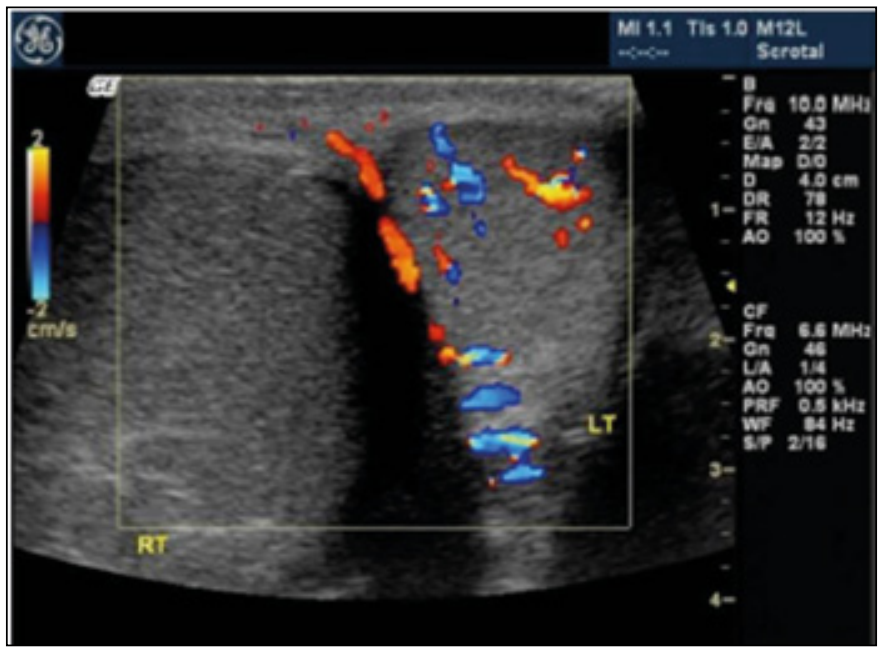

Fig. 7 - Epididimite. L'epididimo appare aumentato di volume e di ecogenicità ridotta. Si associano piccolo idrocele reattivo ed iperemia al power Doppler.

misto, cistico-solido a livello di parenchima prostatico, con echi interni dovuti alla presenza di materiale necrotico. L'ecografia può rivelarsi di particolare utilità come guida per il drenaggio chirurgico di tali raccolte ascessuali. La presenza di piccole formazioni spiccatamente iper-ecogene si identifica, generalmente, con la presenza di calcificazioni di significato benigno.

Suddivisa, secondo McNeal, la prostata in 3 zone, periferica (75\% della gh. prostatica), transazionale e centrale, si osserva come le neoplasie tendano a localizzarsi più frequentemente nella zona periferica $(14,15)$. Al contrario, la sede predominante di sviluppo dell'IPB, è rappresentata dalla zona di transizione.

Le lesioni prostatiche maligne appaiono di solito ipoecogene o tenuemente iperecogene. La presenza di vascolarizzazione, visualizzabile al color ed al power-Doppler è considerata segno di malignità (16), seppure il ruolo diagnostico del Doppler nell'ecografia prostatica sia ancora da definire. Sono invece incoraggianti gli studi che riguardano l'uso del mezzo di contrasto ecografico nel corso dell'esecuzione della biopsia prostatica (17).

\section{L'ecografia nella valutazione dello scroto}

Lo sviluppo di trasduttori ad alta risoluzione per "piccole parti” (7.5-10 MHz) con sistema a circuito liquido incorporato, e l'utilizzo sempre più estensivo del colorpower-Doppler (Aaron F. Wittenberg, 2006) consentono un esame esaustivo del contenuto scrotale, tale da documentare eventuali tumefazioni intra e/o extra-testico-

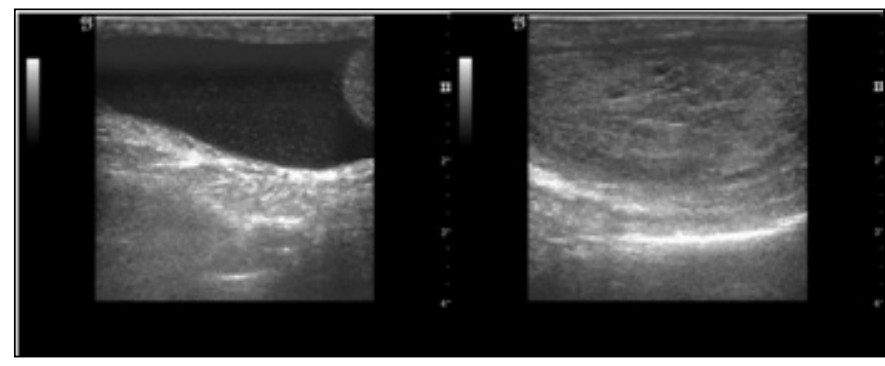

Fig. 8 - Voluminoso idrocele in corso di orchite. Si evidenziano raccolta liquida ipo-ecogena in sede polare inferiore e plurime micro-aree ipo-ecogene intra-testicolari.

lari, solide, cistiche o complesse, esaminare il volume e l'ecogenicità dell'epididimo e l'eventuale presenza di raccolte liquide peri-testicolari.

Il testicolo normale dell'adulto si presenta di forma ovoidale (con diametro massimo di 3-5 cm) con parenchima ad ecogenicità omogenea. Ogni testicolo è circondato da due tuniche, la vaginale, derivata dal peritoneo, e l'albuginea, la quale rappresenta la capsula fibrosa che avvolge il testicolo. Dall'albuginea si dipartono setti, provvisti di circolazione ematica e linfatica, che suddividono il testicolo in 200-300 lobuli. Ciascun lobulo contiene 2-3 tubuli seminiferi, il cui lume si connette con quello della rete testis, e si continua nei dotti efferenti.

Ecograficamente è facile individuare il mediastinum testis (proiezione intra-testicolare della tunica albuginea) che si presenta come una stria lineare iperecogena, nel contesto del quale decorrono i vasi testicolari. $\mathrm{E}$, inoltre, possibile localizzare alcuni residui embrionali come l'idatide di Morgagni, impiantata sul polo superiore del testicolo e l'appendice dell'epididimo (persistenza dell'estremità cranica del condotto mesonefrico). L'epididimo è situato sul versante posteriore del testicolo, con la testa situata posteriormente e superiormente. La sua ecogenicità è in genere omogenea, e la testa, che può misurare fino a $15 \mathrm{~mm}$ è leggermente iper-riflettente rispetto al corpo che, in genere, non supera i $4 \mathrm{~mm}$ di spessore. Nel contesto del parenchima testicolare è possibile documentare la presenza di foci iper-ecogeni puntiformi ("microlitiasi testicolare") in assenza di altre alterazioni della struttura del parenchima circostante $(18,19)$. All'esame istologico, si tratta di piccole calcificazioni dei tubuli seminiferi.

La microlitiasi testicolare può essere bilaterale ed associarsi a criptorchidismo, infertilità, sindrome di Klinefelter, atrofia e neoplasie testicolari $(20,21)$.

In una percentuale pari allo $0.3 \%$ dei soggetti di sesso maschile, soprattutto ragazzi, un testicolo può essere ri- 
tenuto nella regione del canale inguinale o persino in addome. Il "testicolo ritenuto" presenta, solitamente, una struttura parenchimale ecograficamente omogenea, solitamente ipoecogena rispetto a quella di un testicolo normale. Sovente, i testicoli ritenuti in sede addominale, a differenza di quelli ritenuti nel canale inguinale, non sono documentabili ecograficamente (a meno che non coesista una tumefazione di dimensioni apprezzabili), in quanto soggetti ad atrofia. In alternativa essi possono apparire di modeste dimensioni.

Nei testicoli ritenuti il rischio di sviluppare neoplasia è 22 volte maggiore rispetto ai testicoli normalmente discesi (48.9/100 000 contro 2.2/100 000) (22). La ragione di tale incidenza va probabilmente ricercata nell'alterato processo embriogenetico causato dall'elevata temperatura endo-addominale rispetto allo scroto. Occorre, inoltre, considerare l'eventuale coesistenza di squilibri dell'assetto ormonale.

Le neoplasie testicolari rappresentano la classe di tumori più frequenti nei soggetti di sesso maschile con età compresa tra i 20 e i 35 anni. L'ecografia presenta una sensibilità prossima al $100 \%$ nell'identificazione di tali masse (23), le quali si configurano, generalmente, come zone ipoecogene ben delimitate, dotate o meno di una pseudo-capsula (24) e contenenti al loro interno depositi di calcio (documentabili come coni d'ombra in maniera simile a quanto si osserva in corso di nefrolitiasi). In alcuni casi specifici, come le neoplasie a cellule di Leydig (forme tumorali ad elevata aggressività), il quadro ecografico può evidenziare la presenza contemporanea di aree ipo- ed iper-ecogene.

I seminomi si presentano, ecograficamente, con un pattern più omogeneamente ipo-ecogeno, mentre le forme non seminomatose presentano un aspetto più eterogeneo (25).

Le neoplasie testicolari si associano, generalmente, alla presenza di raccolte liquide (idroceli) di piccole dimensioni; tale reperto può generare problemi di diagnosi differenziale, dato che l'idrocele si accompagna spesso a fenomeni di tipo infettivo-infiammatorio (epididimite, orchite) e con la torsione del testicolo (26).

Un linfoma può presentarsi come tumefazione dolente dello scroto, associata alla presenza di linfonodi inguinali palpabili e testicoli aumentati di volume ed ecograficamente disomogenei (più raramente con ecostruttura ipo-ecogena) (27). Si tratta, in genere, di pazienti anziani nei quali sono improbabili fenomeni di torsione testicolare. In questa popolazione di pazienti, la presenza di formazioni intra-testicolari ipo-ecogene, orienta verso la diagnosi di linfoma ovvero di ripetizioni metastatiche. In caso di trauma scrotale, l'ecografia può evidenziare quadri di differente gravità, dalla frattura testicolare, alla temporanea deformità del testicolo, a deficit di vascolarizzazione, contusioni parenchimali, ematoceli ed ematomi scrotali.

L'ematoma da trauma testicolare può portare alla formazione di una zona ipo-ecogena, ben circoscritta all'interno del testicolo (28) con frequente riscontro di falda liquida peri-testicolare.

Aspetti indicativi di frattura scrotale sono le zone focali di abnorme ecogenicità dovute ad emorragia e/o infarto; meno frequentemente è presente una vera e propria linea di frattura ecograficamente evidenziabile (riconducibile alla rottura della tunica albuginea).

Gli ematoceli presentano echi di basso livello, sepimenti, loculi, livelli detrito-liquido, che li differenziano agevolmente dall'idrocele (29).

La maggior parte delle aree ipo-ecogene focali dei testicoli, non associate ad aumento delle dimensioni dell'epididimo e/o alla presenza di un'evidente raccolta liquida, sono indicative di lesioni maligne (30). Nei casi in cui la presenza di un'area ipo-ecogena si associa ad aumentate dimensioni dell'epididimo e concomitante raccolta fluida peri-lesionale, la diagnosi più probabile è quella di epididimite, orchite o torsione testicolare. Lesioni intratesticolari iper-ecogene sono più probabilmente benigne e possono anche configurarsi come extratesticolari (vedi lipomi della corda spermatica, cicatrici fibrose ovvero neoplasia adenomatoide).

In caso di torsione, il testicolo può presentare aree ipoecogene compatibili con ischemia, mentre l'epididimo può essere aumentato di volume ed è possibile riscontrare versamento peri-testicolare; il testicolo controlaterale si presenta invece normale. Il testicolo coinvolto presenta assenza di perfusione all'esame Doppler (31, 32) e, nel caso di torsione cronica, può apparire ipotrofico, povero di echi e con epididimo aumentato di dimensioni (33).

L'ecografia gioca oggi un ruolo essenziale nell'inquadramento diagnostico della torsione, che rappresenta una emergenza chirurgica e necessita di trattamento entro sei ore dalla sua insorgenza (34). In caso di lesione datata può permanere un focus ipo-ecogeno, espressione della cicatrice derivante dalla pregressa torsione, che può simulare una neoplasia.

Strutture cistiche intra-testicolari, esito di infezioni contenenti materiale viscoso e/o emorragico possono presentarsi come lesioni circoscritte ipo-ecogene con echi di basso livello ed essere scambiate per lesioni solide (35). Tale anormalità benigna può essere descritta come cisti epidermoide (unico tumore a cellule germinali benigno) (36); tali formazioni possono presentarsi anche bilaterali e/o multiple.

Nell'epididimite, l'epididimo può presentarsi diffusa- 
mente aumentato di volume, con ecogenicità ridotta e associato a piccolo idrocele reattivo (di entità variabile) ed iperemia al power Doppler. L'epididimite cronica, invece, solitamente si associa a fibrosi ed iperecogenicità. Nell'orchi-epididimite, anche il testicolo può presentare aumento del volume ed ipoecogenicità. L'aspetto è spesso striato o persino disomogeneo, in concomitanza con un aumento dimensionale dell'epididimo, idrocele infiammatorio ed edema cutaneo. In caso di evoluzione ascessuale, l'aspetto ecografico può simulare quello di una neoplasia (37).

Raramente le formazioni extratesticolari presentano caratteristiche di malignità, anche quando queste appaiono iper-ecogene; tipicamente, il testicolo può presentarsi con un versamento associato. La presenza di raccolta liquida di maggiore entità viene definita idrocele e ha solitamente aspetto anecogeno. Tale condizione può avere una causa aspecifica (idrocele semplice) o può essere secondario ad infezioni, neoplasie ovvero trau$\mathrm{mi}$. Se cospicuo, la presenza di un idrocele può rendere impossibile un adeguato esame fisico scrotale rendendo indispensabile l'esame ecografico (38). L'appendice dell'epididimo è ecograficamente più evidente in presenza di idrocele, mentre la loculazione del liquido peritesticolare indica in genere la presenza di un'infezione concomitante (39). Quando i loculi contengono sangue in conseguenza di un trauma, è possibile documentare la presenza di detriti provocata dalla stratificazione dei coaguli ematici.

Lospermatocele o cisti epididimica, può presentarsi come massa anecogena, ripiena di liquido, posta caudalmente al testicolo, che appare ecograficamente normale.

Spermatoceli e cisti di grosse dimensioni possono simulare idroceli e confondersi anche con varicoceli (40). A differenza di questi, però, gli spermatoceli si sviluppano di solito verso il canale inguinale fino a deformare il testicolo. Il varicocele (dilatazione venosa del plesso pampiniforme) si configura come voluminosa area multiloculare ipo-anecogena, il cui aspetto potrebbe modificarsi alla manovra di Valsalva, adiacente al testicolo di aspetto normale (41). Il color Doppler ed il power Doppler riveleranno la natura vascolare della lesione, confermando la diagnosi e l'indicazione alla rivalutazione chirurgica del paziente, dati i noti potenziali effetti deleteri della patologia sulla fertilità.

\section{Riassunto}

Le infezioni delle vie urinarie costituiscono un problema clinico frequente in tutte le età. L'ecografia è uno strumento prezioso nella diagnosi e nel management delle infezioni, consentendo di diagnosticare rapidamente complicanze e fattori predisponenti.

Questa review passa in rassegna gli aspetti ecografici delle infezioni della vescica, della prostata, delle vescicole seminali, dei didimi, degli epididimi e dello scroto, fornendo un pratico strumento di consultazione rapida al nefrologo.

Parole Chiave. Ecografia, Infezioni delle vie urinarie, Cistite, Prostatite, Orchite

\section{Indirizzo degli Autori: \\ Dr. Antonio Granata \\ Via F. Paradiso 78/a \\ 95024 Acireale (CT) \\ antonio.granata4@tin.it}

\section{Bibliografia}

1. Hooton TM. UTIs: An Overview. The epidemiology of urinary tract infection and the concept of significant bacteriuria. Infection18: S40-S43.

2. Vick CW, Viscomi GN, Mannes E, Taylor KJ. Pitfalls related to the urinary bladder in pelvic sonography: a review. Urol Radiol 1983; 5(4): 253-9.

3. Laing FC, Kurtz AB. The importance of ultrasonic sidelobe artifacts. Radiology 1982; 145(3): 763-8.
4. Johansen TE. The role of imaging in urinary tract infections. World J Urol 2004; 22(5): 392-8. Epub 2004 Jul 29.

5. Bjerklund Johansen TE. Diagnosis and imaging in urinary tract infections. Curr Opin Urol 2002; 12(1): 39-43.

6. Quint HJ, Drach GW, Rappaport WD, Hoffmann CJ. Emphysematous cystitis: a review of the spectrum of disease. J Urol 1992; 147(1): 134-7.

7. Czaja CA, Stamm WE, Stapleton AE, et al. Prospective cohort study of microbial and inflammatory events immediately preceding Escherichia coli recurrent urinary tract 
infection in women. J Infect Dis 2009; 15: 200(4): 528-36.

8. Hanno P, Nordling J, Fall M. Bladder pain syndrome. Med Clin North Am 2011; 95(1): 55-73.

9. Najar MS, Saldanha CL, Banday KA. Approach to urinary tract infections. Indian J Nephrol 2009; 19(4): 129-39.

10. Berkey FJ. Managing the adverse effects of radiation therapy. Am Fam Physician 2010; 15; 82(4): 381-8, 394.

11. Benson MC, Whang IS, Olsson CA, McMahon DJ, Cooner $\mathrm{WH}$. The use of prostate specific antigen density to enhance the predictive value of intermediate levels of serum prostate specific antigen. J Urol 1992; 147(3 Pt 2): 817-21.

12. Hodge KK, McNeal JE, Terris MK, Stamey TA. Random systematic versus directed ultrasound guided transrectal core biopsies of the prostate. J Urol 1989; 142(1): 71-4; discussion 74-5.

13. Kabalin JN, Hodge KK, McNeal JE, Freiha FS, Stamey TA. Identification of residual cancer in the prostate following radiation therapy: role of transrectal ultrasound guided biopsy and prostate specific antigen. J Urol 1989; 142(2 Pt 1): 326-31.

14. McNeal JE. The zonal anatomy of the prostate. Prostate 1981; 2(1): 35-49.

15. McNeal JE. Anatomy of the prostate and morphogenesis of BPH. Prog Clin Biol Res 1984; 145: 27-53.

16. Rifkin MD, Sudakoff GS, Alexander AA. Prostate: techniques, results, and potential applications of color Doppler US scanning. Radiology 1993; 186: 509-13.

17. Halpern EJ, Rosenberg M, Gomella LG. Prostate cancer: contrast-enhanced us for detection. Radiology 2001; 219: 219-25.

18. Pourbagher MA, Kilinc F, Guvel S, Pourbagher A, Egilmez T, Ozkardes H. Follow-Up of Testicular Microlithiasis. Urol Int 2005; 74: 108-13; DOI: 10.1159/000083279.

19. Bushby LH, Miller FN, Rosairo S, Clarke JL, Sidhu PS. Scrotal calcification: ultrasound appearances, distribution and aetiology. Br J Radiol 2002; 75: 283-8.

20. Kocaoğlu M, Bozlar U, Bulakbaşı N, Sağlam M, Üçöz $\mathrm{T}$, Somuncu I. Testicular microlithiasis in pediatric age group: ultrasonography findings and literature review. Diagn Interv Radiol 2005; 11: 60-5.

21. Cast JE, Nelson WM, Early AS, et al. Testicular Microlithiasis: Prevalence and Tumor Risk in a Population Referred for Scrotal Sonography. AJR 2000; 175: 1703-06.

22. Gamal El-Husseiny M, Shouki BazarbashiA. Amr ElWeshi D, Mahmoud EL-Foudeh MM, Amjad Perracha FA. Germ Cell Tumors in Undescended Testis-Prognostic Factors and Treatment Outcome. Journal of the Egyptian Nat. Cancer Inst 2001; 13(3): 209-14.

23. Gray scale and doppler ultra-sound in the diagnosis of painless scrotal masses. Pak J Med Sci 2010; 26(1): $178-82$.
24. Florentine BD, Roscher AA, Garrett J, Warner NE. Necrotic seminoma of the testis: establishing the diagnosis with Masson trichrome stain and immunostains. Archives of Pathology \& Laboratory Medicine 2002, Feb 1.

25. Liu S, Semenciw R, Waters C, Wen SW, Mery LS, MaoY. Clues to the aetiological heterogeneity of testicular seminomas and non-seminomas: time trends and age-periodcohort effects. Int J Epidemiol 2000; 29: 826-31.

26. Trojian TH, Lishnak TS, Heiman D. Epididymitis and Orchitis: An Overview. Am Fam Physician 2009; 79(7): 583-7.

27. Mazzu D, Jeffrey RB, Ralls PW. Lymphoma and Leukemia Involving the Testicles: Findings on Gray-Scale and and Color Doppler Sonography. AJR 164: 645-7.

28. The painful scrotum: an ultrasonographical approach to diagnosis. Singapore Med J 2005; 46(7): 352-7.

29. Gerscovich EO. (1993). High-resolution ultrasonography in the diagnosis of scrotal pathology: I. Normal scrotum and benign disease. J Clin Ultrasound 1993; 21(6): 355-73.

30. ismail Mihmanlı FK. Sonography of scrotal abnormalities in adults: an update. Diagnostic and Interventional Radiology 2009; 15: 64-73.

31. Şanlı Y, Adalet I, Handan Tokmak H, Şanlı Ö, Ziylan O, Cantez S. Radionuclide imaging in differential diagnosis of torsion and infections of testis and epidymis revisited. Marmara Medical Journal 2006; 19(3): 132-4.

32. Anand Brig SS, Mukherjee PB, Kumar PG, Singh J. Scrotal Scintigraphy in a Case of Acute Scrotum. MJAFI 2008; 64: $92-4$.

33. Maj RE, Jesinger JB. Testicular torsion. Military Medicine Radiology Corner 2009; 174.

34. Ringdahl E, Teague L. Testicular Torsion. Am Fam Physician 2006; 74(10): 1739-43.

35. Shergill IS, Thwaini A, Kapasi F, Potluri BS, Barber C. Management of simple intratesticular cysts: a single-institution 11-year experience. Urology 2006; 67 (6): 1266-8.

36. Harkin DW, Pyper P, Hawe M. Testicular epidermoid cyst: a case for conservative surgery. The Ulster Medical Journal 1998; 67(2): 142-4.

37. Necrotising epididymo-orchitis with scrotal abscess. Biomed Imaging Interv J 2005; 1(2): e11 .

38. Ultrasound features of spermatic cord hydrocele in children. The British Journal of Radiology 2001; 74: 818-20.

39. Carroll BA, Gross DM. High Frequency scrotal sonography. AJR Am J Roentgenol 1983; 140: 511-5.

40. E Huri, Akgul T, Ayyildiz A, Germiyanoglu C. Bilateral giant spermatocele mimicking an intrascrotal mass. Turkish Journal of Urology 2009; 35(2): 151-2.

41. Williams DH, Karpman E, Lipshultz LI. Varicocele: surgical techniques in 2005. Can J Urol 2006; 10 (Suppl 1), S19-23. 